\title{
ELECTROPHORESIS OF RANDOMLY ORIENTED CYLINDRICAL PARTICLES
}

\author{
A. DE KEIZER, W.P.J.T. VAN DER DRIFT and J.Th.G. OVERBEEK \\ Van 't Hoff Laboratory for Physical and Colloid Chemistry, Transitorium III. \\ Padualaan 8, University of Utrecht, The Netherlands
}

Received 19 September 1974

Revised manuscript received 7 November 1974

It has been derived, that the electrophoretic mobility of a randomly oriented charged cylinder is obtained by adding onethird of the mobility of a cylinder parallel to the field to two-thirds of its mobility perpendicular to the field, when the relaxation effect is neglected.

The calculation of the electrophoretic mobility of randomly oriented charged cylinders is of special interest, because it may be applied in an approximate way to molecules of biological importance such as DNA and to other linear poly-electrolytes. The average electrophoretic mobility of randomly oriented cylinders is found by taking a suitable average of the mobilities of a cylinder with its axis perpendicular to the electric field, $(U / X)_{L}$ and of one parallel to the field, $(U / X)_{\|}$. Remarkably enough, even in the recent literature two different methods of averaging can be found, one method averaging mobilities [ $1-3$ ], the other one averaging reciprocal mobilities $[4,5]$. It is the purpose of this paper to prove that, when the relaxation effect is neglected, the correct result is obtained by averaging mobilities.

For nonconductìng cylinders, Henry [6] has given the following equations:

$$
\begin{aligned}
& \left(\frac{U}{X}\right)_{\| l}=\frac{\epsilon \psi_{a}}{4 \pi \eta}, \\
& \left(\frac{U}{X}\right)_{\perp}=\frac{\epsilon \psi_{a}}{\pi \eta F(k a)} .
\end{aligned}
$$

where $a$ is the radius of the cylinder, $\psi_{a}$ the electrostatic potential at its surface, $1 / k$ the thickness of the electric double layer, $\epsilon$ the dielectric constant and $\eta$ the viscosity of the medium.

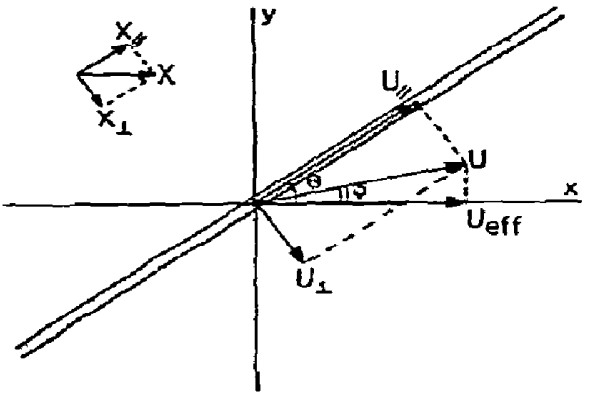

Fig. 1. An arbitrarily oriented charged cylinder with electrophoretic velocity $U$, in an electric field with field strength $\boldsymbol{X}$.

The function $F(k a)$ can be calculated according to Henry $[6,7]$. It has values between 4 and 8 .

Fig. 1 shows an arbitrarily oriented cylinder. The electric field is along the $x$-axis. The cylinder makes an angle $\theta$ with the field $\left(0 \leqslant \theta \leqslant 90^{\circ}\right)$.

Instead of solving the electrical and hydrodynamic equations for this arbitrary orientation, we can find the correct solution for the total electric field and the total hydrodynamic flow by vectorial addition of the field and the flow caused by the components of the applied field parallel and perpendicular to the cylinder axis respectively*. Since $(U / X)_{\|}$and $(U / X)_{\perp}$ are not

* It may readily be shown that the splitting up of the hydrodynamic differential equations in the two mentioned independent sets is correct. if one neglects some of the inertia terms in the Navier-Stokes equation [6]. 
identical the electrophoretic velocity of the cylinder is in general not in the direction of the freld, but it makes an angle, $\varphi$, with it. The observable velocity,

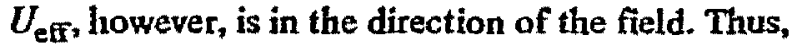
using eqs. (1) and (2) we obtain

$U_{\text {eff }}=U \cos \varphi=U_{\|} \cos \theta+U_{\perp} \sin \theta$

$=\frac{\epsilon \psi_{a}}{\pi \eta}\left(\frac{X_{\mathrm{I}} \cos \theta}{4}+\frac{X_{1} \sin \theta_{4}}{F(\kappa a)}\right)=\frac{\epsilon \psi_{a} X}{m}\left(\frac{\cos ^{2} \theta}{4}+\frac{\sin ^{2} \theta}{F(\kappa a)}\right)$.

[It is interesting to notice, that $\varphi$ has a maximum of $19^{\circ} 28^{\prime}$ if $\theta=55^{\circ}$ for $F(\mathrm{ka})=8$.] The average electrophoretic velocity is obtained by integrating $U_{\text {eff }}$ over all possible orientations.

$\left\langle U_{\mathrm{eft}}\right\rangle=\int_{0}^{\frac{1}{2} \pi} U_{\mathrm{efr}} \sin \theta \mathrm{d} \theta=\frac{1}{3} \frac{\varepsilon X \psi_{a}}{\pi}\left(\frac{1}{4}+\frac{2}{F(\kappa a)}\right)$,

or

$\left\langle U_{\text {eff }} / X\right\rangle=\frac{1}{3}\left[(U / X)_{1]}+2(U / X)_{\perp}\right]$

in agreement with refs. [1-3] but different from the incorrect result $\left(x / U_{\text {eff }}\right)=\frac{1}{3}\left[(x / U)_{4}+2(x / U)_{1}\right]$

as used in refs. [4] and [5].

The derivation given here does not apply anymore when the distortion of the double layer by the relaxation effect has to be taken into account, because then end effects due to the finite length of the cylinder can no longer be neglected.

\section{References}

[1] J.Th.G. Overbeek, in: Advances in colloid science, Vol. III, eds. H. Mark and E.J.W. Verwey (Interscience, New York, 1950).

[2] J.Th.G. Overbeek and P.H. Wiersema, in: Electrophoresis, Vol. II, ed. M. Bier (Academic Press, London, 1967).

[3] T. Takahashi, I. Noda and M. Nagasawa, J. Phys. Chern. $74(1970) 1280$.

[41 H.A. Abramson, LS. Moyer and M.H. Gorin, Electrophoresis of proteins (Reinhold, New York, 1942).

[5] R.A. Mills, Biopolymers 9 (1970) 1511.

[6] D.C. Henry, Prac. Roy. Soc. Al33 (1931) 106.

[7] W.P.J.T. van der Drift, A. de Keizer and J.Th.G. Overbeek, paper in preparation. 